\title{
Conceptos para la cuantificación del consumo estructural y su aplicación a estructuras generadas por elementos preflectados
}

\author{
Structural consumption measure concepts and their application \\ to structures generated by bending-active elements
}

A. García Santabárbara ${ }^{(*)}$, J. Cervera Bravo ${ }^{(*)}$

RESUMEN

En el ámbito del proceso de toma de decisiones propio del proyecto de estructuras resistentes, el mero análisis mecánico de un modelo no permite vislumbrar las posibles disminuciones de material derivadas de modificaciones en la forma estructural. Dado que para cuantificar estas mejoras es imprescindible poder medir el consumo estructural ya en las primeras fases de diseño, en el presente trabajo se presentan y comparan dos de las teorías actualmente más desarrolladas que relacionan la geometría con el consumo estructural. Para demostrar la versatilidad de estos conceptos se aplicarán a continuación a un tipo de estructuras que cuenta simultáneamente con esfuerzos internos previos y grandes deformaciones: las estructuras emparejadas, un caso particular de estructuras generadas por elementos preflectados, en los que los componentes se asocian dos a dos para formar grupos arco-tirante.

Palabras clave: consumo estructural; optimización; estructura preflectada; volumen de tensiones; cantidad de estructura; Bic-lambda.

\section{ABSTRACT}

In the sphere of decision-making for resistant structures project, the mere mechanical analysis of a model does not suggest the possible material reduction resulting from changes in the structural form. In order to quantify these improvements it is essential to be able to measure the structural consumption in the early stages of design. So in this paper we present and compare two of the currently most developed theories that connect geometry with material consumption of the structure. To demonstrate the versatility of these concepts, they will be applied to a type of structure with both residual stresses and large deformations: coupled structures. This particular case of structures is generated by pre-bent elements, where the components are associated by pairs to form tied-archs groups.

Keywords: structural efficiency; optimization; bending-active structure; stress volume; quantity of structure; Biclambda.

(*) Escuela Técnica Superior de Arquitectura - Universidad Politécnica de Madrid, Madrid (España). Persona de contacto/Corresponding author: alfonsogarsan@gmail.com (A. García Santabárbara) ORCID: http://orcid.org/oooo-0o01-9176-0950 (A. García Santabárbara); http://orcid.org/oooo-00o2-1060-7397 (J. Cervera Bravo)

Cómo citar este artículo/Citation: García Santabárbara, A., Cervera Bravo, J. (2017). Conceptos para la cuantificación del consumo estructural y su aplicación a estructuras generadas por elementos preflectados. Informes de la Construcción, 69(546): e201, doi: http:// dx.doi.org/10.3989/ic.16.016.

Copyright: (C) 2017 CSIC. Licencia / License: Salvo indicación contraria, todos los contenidos de la edición electrónica de Informes de la Construcción se distribuyen bajo una licencia de uso y distribución Creative Commons Attribution License (CC BY) Spain 3.o. 


\section{INTRODUCCIÓN}

Si definimos eficiencia como la capacidad para llevar a cabo un fin empleando la menor cantidad de recursos, parece lógico pensar que, en principio, ésta debería ser el principal objetivo del responsable de la concepción de estructuras resistentes. Sin embargo, en las primeras fases del proceso proyectual la mayoría de decisiones van encaminadas hacia la definición geométrica de la propuesta y el abanico de posibilidades formales es todavía lo suficientemente amplio como para no permitir un análisis exhaustivo de cada modelo. Existe, por lo tanto, una necesidad real de conceptos que vinculen forma y eficiencia estructural. Estos conceptos se convertirían en útiles herramientas que nos permitirían cualificar o comparar estructuras en las fases iniciales, sin que fuera necesario desarrollar con precisión las propuestas ni realizar un dimensionado riguroso.

Tal es el valor de las relaciones entre los parámetros geométricos y las capacidades mecánicas que hasta mediados del siglo XVII se establecieron como el principal criterio para avalar el éxito de un modelo estructural. Una vez probada la viabilidad constructiva de un modelo mediante la práctica, la extrapolación al resto de tamaños se creía garantizada, siempre y cuando se mantuvieran las proporciones originales. Esta teoría se mantuvo irrefutable hasta la publicación de los Discorsi de Galileo en 1638 y que supuso la negación, desde la geometría, del libre escalado de los modelos estructurales (1). Los avances en el cálculo diferencial de los siglos posteriores consiguieron que las reglas geométricas quedaran abandonadas casi definitivamente del análisis estructural. Salvo contadas excepciones, entre las que merecen ser citadas explícitamente las de Rankine (2), Maxwell (3) y Michell (4), éste será el escenario que se mantendrá hasta la segunda mitad del siglo XX, cuando la búsqueda de la ligereza se convierta en una cuestión de relevancia para la arquitectura de la época (Wachsmann, Fuller, Prouvé).

Todo proceso de cuantificación objetiva es el resultado de una comparación entre distintos valores numéricos. Por lo que para poder comparar la eficiencia de distintos modelos estructurales se han de poder medir las vinculaciones entre geometría y comportamiento estructural. En el proceso de búsqueda de una magnitud física que incluya ambos conceptos surgen, a mediados del siglo XX y de forma independiente, las dos líneas de investigación del consumo estructural que se van a relacionar aquí: la representada por los trabajos de Frei Otto en el actual Institut für Leichtbau Entwerfen und Konstruieren de la Universidad de Stuttgart y la de Ricardo Aroca al frente del Departamento de Estructuras de la Edificación de la Escuela Técnica Superior de Arquitectura de la Universidad Politécnica de Madrid.

Por algún extraño motivo ambas investigaciones han seguido desarrollos más o menos paralelos, incluso en ocasiones demasiado próximos, sin que existan referencias que los hayan relacionado con anterioridad. El presente trabajo expondrá los principales conceptos de la teoría de consumo material de estructuras de ambas escuelas para realizar una posterior comparación.

Por otro lado, en los últimos cinco años, desde el proyecto del pabellón temporal del ICD/ITKE 2010, se está produciendo una revisión de las estructuras de geometría curva obtenida a partir de la deformación y el ensamblaje de piezas original- mente planas. En este proceso constructivo de preflectado se introducen tensiones internas esperando obtener algún tipo de beneficio estructural, generalmente un aumento de rigidez y/o eficiencia. Para poder cuantificar esta mejora se planteará la aplicación de los conceptos de consumo estructural a este tipo de estructuras.

\section{STUTTGART}

El Institut für Leichte Flächentragwerke (incluido desde 2001 en el Institut für Leichtbau Entwerfen und Konstruieren) edita en 1998 la investigación que Frei Otto, primero de forma individual y posteriormente junto con el grupo de trabajo del IL, ha venido llevando a cabo desde la segunda mitad de la década de los años 40. Otto, motivado por la escasa disponibilidad de material de construcción que provocó la Segunda Guerra Mundial, y como responsable de la reconstrucción de puentes y cubiertas de naves en Le Coudray (cerca de Chartres), define el concepto de «valor de comparación de la construcción ligera» como «la suma de todos los productos de las fuerzas de las barras por las longitudes de éstas en las cerchas» (5).

En esta publicación, además de la identificación de tres conceptos (Tra, Bic y $\lambda$ ) para la cuantificación del consumo estructural, se realiza su aplicación mediante un abundante y exhaustivo estudio gráfico a casi todos los ámbitos en los que es posible su uso, no sólo estructurales, sino también en problemas de transporte o consumo de energía. Todo este trabajo queda resumido en una tabla y un diagrama. La primera (Figura 1) incluye el valor del Tra relativo de las soluciones de estructuras de barras más representativas concepto comparable con el número de Michell de la otra teoría, tal y como se explica más adelante-. El diagrama (Figura 2) abarca las relaciones Bic- $\lambda$-enlazadas con las magnitudes «alcance» $\mathrm{y}$ «esbeltez» de la escuela madrileña- de casi la totalidad de los tamaños y tipos de estructuras. Juntos, tabla y diagrama se convierten en un entorno de valores numéricos de referencia que sirven de orientación para realizar comparaciones directas, utilizando solamente el esquema y tamaño, y, por lo tanto, previas al proceso de dimensionado.

\subsection{Tra}

Otto define el concepto genérico de Tra como medida del transporte de cargas (y de masas). De esta forma el mismo concepto se puede aplicar a tres niveles distintos: sirve para medir la magnitud del problema estructural (Ta, Tra externo), para cuantificar el recorrido de transmisión de fuerzas de un esquema estructural ( $\mathrm{Ti}$, Tra interno) o para medir el desvío de carga que supone la utilización de un modelo u otro (Tr, Tra relativo).

El primero de ellos, Tra externo, se determina mediante el producto de todas las cargas exteriores y la menor distancia de cada carga a la superficie de apoyo, o al apoyo más cercano si éstos son puntuales (Figura 3). Usando siempre valores positivos para las fuerzas, con independencia de la dirección y sentido y ángulo de éstas respecto del recorrido.

$$
\mathrm{Ta}=\sum\left(\mathrm{F}_{\mathrm{a}} \cdot \mathrm{s}_{\mathrm{a}}\right)
$$

En segundo lugar, el Tra interno se calcula mediante el sumatorio de los productos de los esfuerzos internos por la dis- 


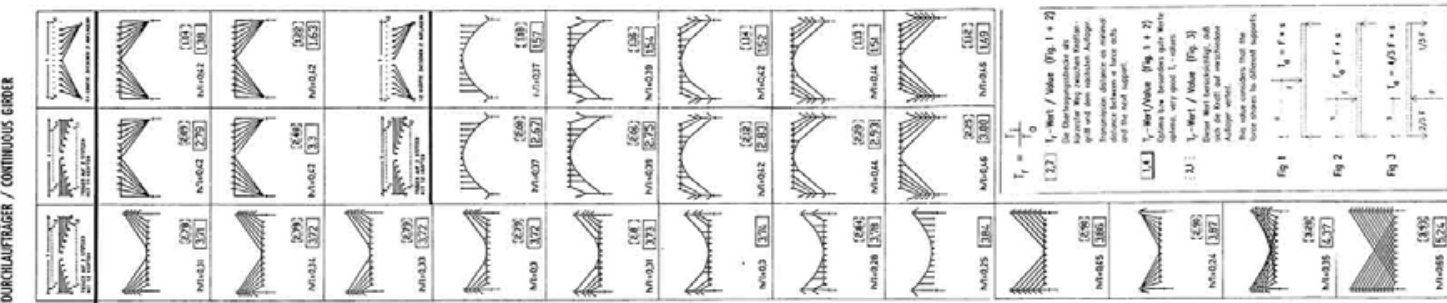

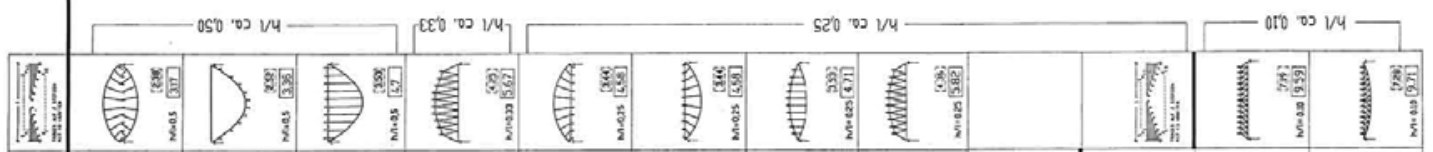

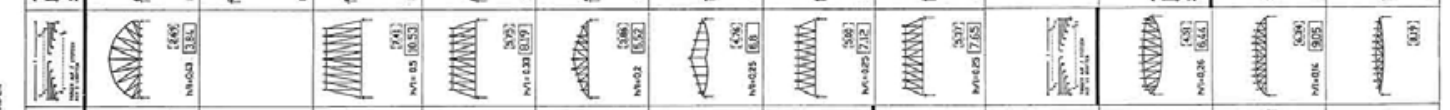

1

H.

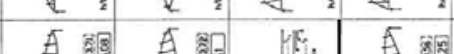

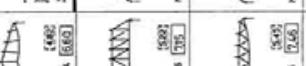

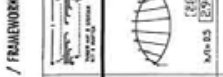

稫部

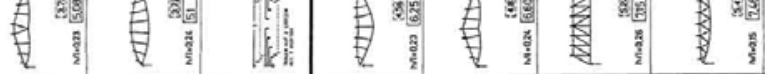

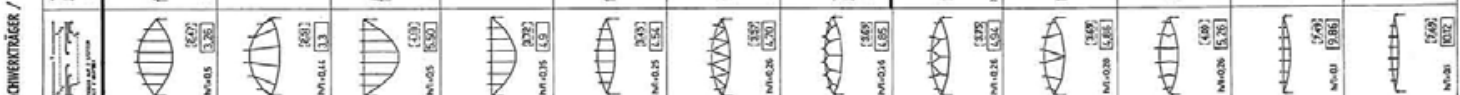

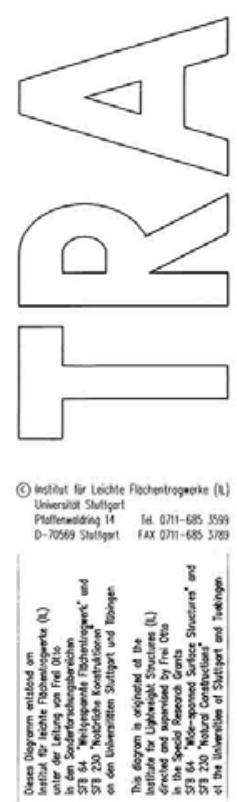

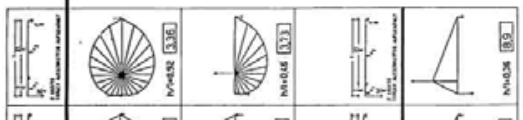

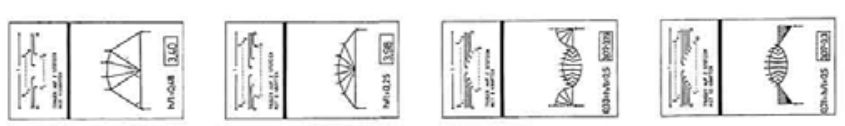
Int
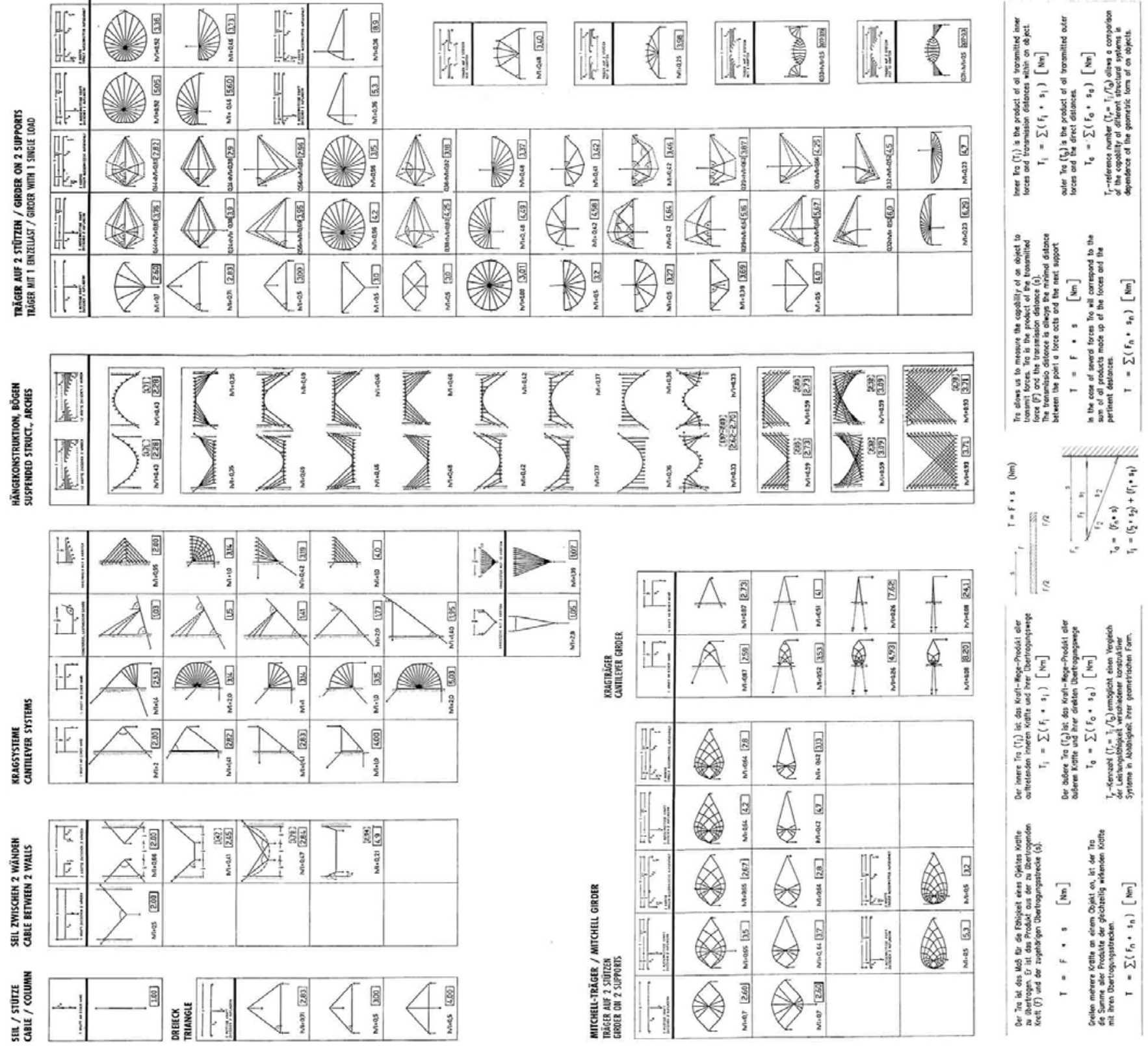

Figura 1. Tabla Tra. Fuente: (5). 

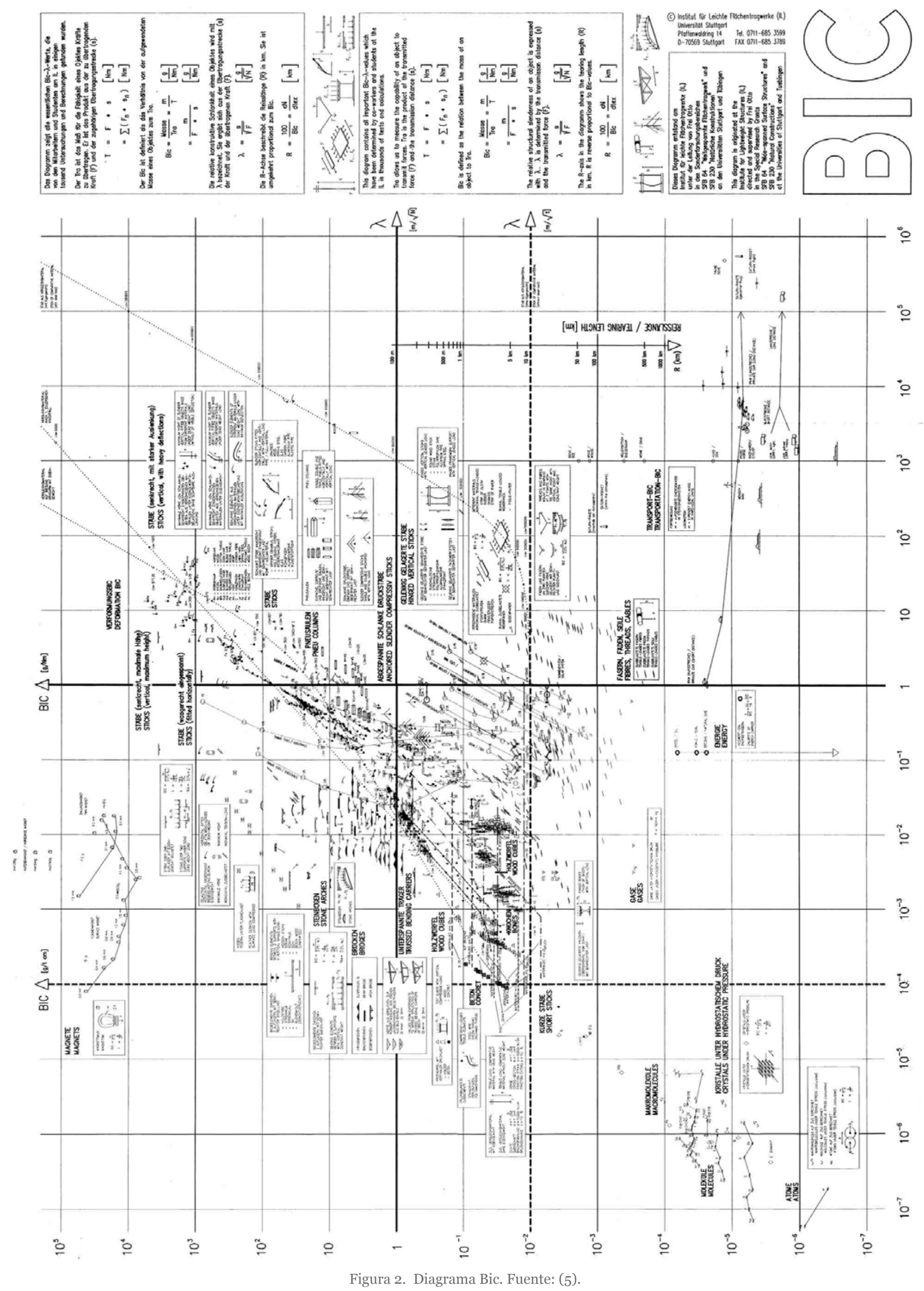


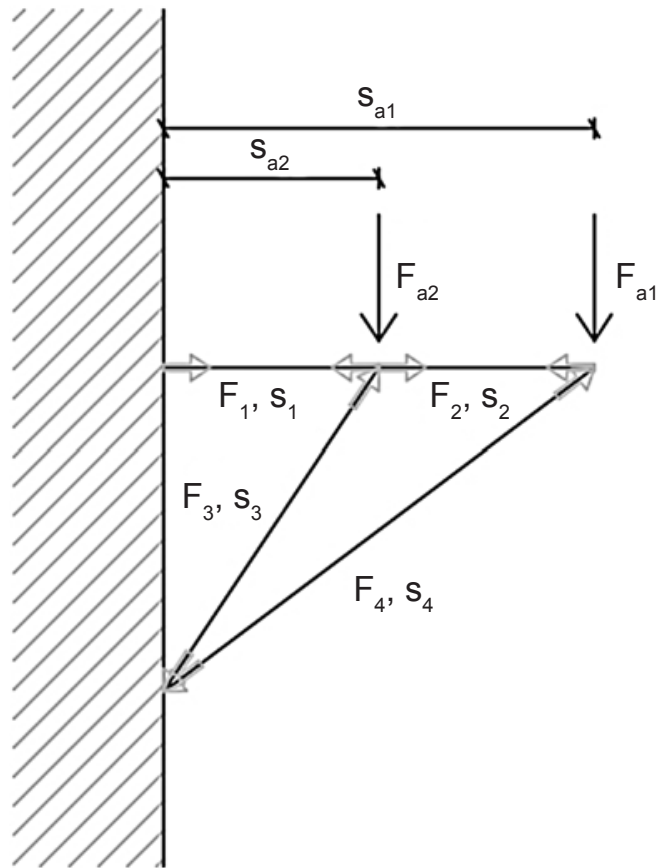

Figura 3. Tra interno y Tra externo.

tancia de la parte de estructura a través de la que se transmiten esos esfuerzos. En el caso de estructuras de barras se obtendría con el sumatorio del producto del esfuerzo por la longitud de cada barra.

$$
\mathrm{Ti}=\sum\left(\mathrm{F}_{\mathrm{i}} \cdot \mathrm{s}_{\mathrm{i}}\right)
$$

Por último, el concepto Tra relativo representa la razón entre la capacidad de transporte de carga del modelo y la magnitud del problema estructural. El valor mínimo absoluto es la unidad y se obtiene para esquemas unidimensionales, cuando Ti y Ta coinciden.

$$
\mathrm{Tr}=\mathrm{Ti} / \mathrm{Ta} \geq 1
$$

\subsection{Bic}

La capacidad para transportar cargas de un modelo estructural queda definida por la magnitud Tra, con independencia del material usado. Consecuentemente, para introducir las propiedades materiales de la estructura se introduce el concepto de Bic, que relaciona el esquema (distancia y fuerza) con la masa. La masa aquí referida es la masa mínima, y por lo tanto utilizada a su máxima capacidad portante o tensión de rotura.

$$
\mathrm{Bic}=\mathrm{m} / \mathrm{Ta}
$$

El concepto de Bic está planteado de tal forma que, para la misma capacidad portante, un valor mayor representa una mayor cantidad de masa necesaria. Por lo que la eficiencia puede medirse como la función inversa de Bic.

Además del Bic para la carga de rotura, Otto plantea otras versiones de Bic. Por ejemplo, para las estructuras en las que se producen grandes desplazamientos (como las estructuras preflectadas) es posible calcular el Bic de deformación, empleando el valor de la masa para la que se produciría una determinada deformación. O lo que llama Bic material, definido como el cociente entre densidad y tensión de rotura. También define el Bic para cargas «vivas», «muertas», de tráfico y energético.

\section{3. $\lambda$, lambda}

No siempre es posible el empleo directo de los conceptos de Tra y Bic en los procesos de optimización estructural, debido a la existencia de casos de transporte de cargas en los que es necesario asegurar la estabilidad de determinados elementos. En los modelos que contienen elementos comprimidos o flectados los problemas de pandeo provocan que el valor de Bic aumente si lo hace la esbeltez. Para incluir la influencia de la forma en el valor de Bic, Otto introduce un último concepto, $\lambda$, que relaciona geometría y fuerza. Por lo tanto, la eficiencia de los modelos estructurales queda definida, de forma genérica, por la pareja Bic- $\lambda$.

$$
\lambda=\mathrm{s} / \sqrt{ } \mathrm{F}
$$

\section{ETSAM}

Las relaciones entre tamaño, forma y proporción en el ámbito de la resistencia de materiales son el tema principal de la investigación iniciada por Ricardo Aroca, a partir de los años sesenta, en el Departamento de Estructuras de la Edificación de la Escuela Técnica Superior de Arquitectura de la Universidad Politécnica de Madrid y que profesores como José Luis de Miguel (6), Juan Antonio González Cárceles (7), Jaime Cervera (8) (9) (10), José Luis Fernández Cabo (1) y Mariano Vázquez Espí (9) (10) han continuado.

Tanto en las tesis doctorales como en las publicaciones de este grupo de investigación se ha desarrollado con mayor detalle el estudio matemático-diferencial de los conceptos de consumo estructural. Esta evolución teórica permite, en primer lugar, su aplicación a medios continuos, y en segundo lugar, la definición de teoremas y corolarios de aplicación directa durante la fase de diseño.

\subsection{Cantidad de estructura y Número de Maxwell}

El concepto base que permite relacionar matemáticamente forma y comportamiento estructural es el denominado cantidad de estructura, W. Éste representa a la integral extendida a toda la estructura de los productos $|\mathrm{N}| \cdot \mathrm{ds}$ siendo $\mathrm{N}$ el esfuerzo axial en la «sección» y ds el elemento longitudinal de pieza en el punto considerado, y donde $\mathrm{N}>0$ implica tracción y $\mathrm{N}<$ o compresión.

$$
\mathrm{W}=\int|\mathrm{N}| \cdot \mathrm{ds}
$$

Dado que el término de integración es lineal, es posible desglosar la cantidad de estructura total en las partes que mejor convengan para el análisis del modelo, como comprimida y traccionada, vertical y horizontal, o cantidad de estructura por elementos, como cordones y alma, por ejemplo (Figura 4).

La relación entre cantidad de estructura y volumen de ésta es inmediata en el caso de estructuras «estrictas» (trabajando en todos sus puntos a la máxima tensión compatible con la seguridad), siendo $\mathrm{W}=\sigma \cdot \mathrm{V}$, pues

$$
\mathrm{W}=\int \sigma \cdot \mathrm{A} \cdot \underline{d s}=\int \sigma \cdot \mathrm{dV}
$$

razón por la que también se usa la denominación «volumen de tensiones». 


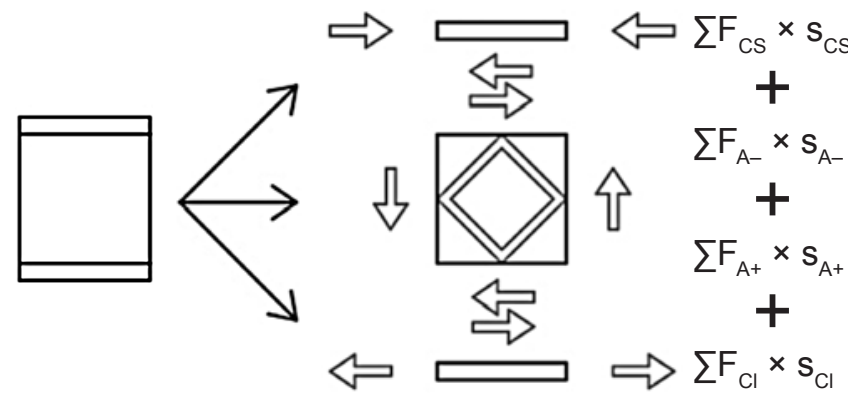

Figura 4. Cantidad de estructura de un sistema como suma de la cantidad de estructura de sus componentes.

\subsection{Teorema de Maxwell o constancia del Número de Maxwell}

$\mathrm{Al}$ introducir el valor absoluto en la expresión de la cantidad de estructura se asegura la suma tanto de tracciones como de compresiones. Este matiz es importante. Para tener en cuenta la influencia del signo de los esfuerzos se introduce un nuevo concepto, el Número de Maxwell, $\mathrm{M}$.

$$
\mathrm{M}=\int \mathrm{N} \cdot \mathrm{ds}
$$

De esta forma, agrupando los elementos traccionados por un lado y elementos comprimidos por el otro, se obtiene que el Número de Maxwell es la diferencia entre la cantidad de estructura traccionada y la cantidad de estructura comprimida.

$$
\begin{gathered}
M=\int \mathrm{N} \cdot \mathrm{ds}=\int \mathrm{N}^{+} \cdot \mathrm{ds}+\int \mathrm{N}^{-} \cdot \mathrm{ds}=\int \mathrm{N}^{+} \cdot \mathrm{ds}-\int\left|\mathrm{N}^{-}\right| \cdot \mathrm{ds} \\
\mathrm{M}=\mathrm{W}^{+}-\mathrm{W}^{-}
\end{gathered}
$$

En el conocido como «lema (o teorema) de Maxwell» (3), este científico demostró que dicha magnitud es función únicamente de las fuerzas aplicadas y de los puntos de aplicación, por lo que es independiente de la forma de la estructura. Siempre y cuando las condiciones del problema estructural se mantengan inalterables, el valor del Número de Maxwell es idéntico para las distintas soluciones posibles (8).

Si la diferencia entre cantidad de estructura traccionada y comprimida se mantiene constante, la solución que anule alguno de estos términos es, necesariamente, una estructura mínima. Es decir, las estructuras sólo traccionadas o sólo comprimidas quedan así definidas como mínimas. Asimismo la disminución de una de estas dos partes implica la disminución de la otra, siempre que se continúe resolviendo el mismo problema estructural.

\section{3•3. Número de Michell}

El número de Michell es un número adimensional que resulta de dividir la cantidad de estructura de la solución propuesta y un valor de referencia. Este valor de referencia representa la intensidad del problema estructural, y se obtiene como el producto de la carga total y la dimensión representativa del problema que la estructura resuelve (luz en flexión y altura para tracción o compresión). El valor así obtenido para el número de Michell es el inverso de la eficiencia, lo que permite destinarlo a establecer comparaciones entre los distintos modelos alternativos.

$$
\mathrm{v}=\mathrm{W} /(\mathrm{Q} \cdot \mathrm{l})
$$

\subsection{Esbeltez}

En la caracterización de la «cantidad de estructura» en problemas de flexión la formulación generalizada queda de la siguiente forma:

$$
\mathrm{W}=\gamma \mathrm{Ql} \lambda
$$

Donde además de los términos Q y l aparecen en la expresión la esbeltez y un factor, $\gamma$, que remite a la forma del modelo en su sentido más general, pues es dependiente del tipo de carga, de la forma de la estructura en las inmediaciones del apoyo, de la geometría de la sección, etc., pero también levemente de la esbeltez, siendo muy estable para las esbelteces habituales. En sentido estricto, depende de la unidad más el cuadradado del cociente entre esbeltez óptima y esbeltez real (9). Por comparación entre [8] y [9] se ve que el Número de Michell es $v=\gamma \lambda$.

Por otro lado, en los problemas de compresión la medida del efecto de la mayor o menor intensidad de la carga -que implica esbeltez, y el correspondiente sobredimensionado por pandeo- se realiza a través del concepto introducido por Aroca y de Miguel, $\mathrm{l}^{2} / \mathrm{N}$.

\section{ANÁLISIS COMPARATIVO DE AMBAS TEORÍAS}

En esta sección se presenta un análisis comparativo entre las dos teorías de consumo estructural previamente estudiadas. Las coincidencias y divergencias son las siguientes:

a) La caracterización de los modelos estructurales se realiza en ambas teorías mediante un escalar, independiente de la dirección y el sentido de las cargas. Ni el concepto de Tra ni el de cantidad de estructura tienen en cuenta estos parámetros, permitiendo establecer comparaciones entre estructuras comprimidas, traccionadas y flectadas.

b) La formulación de Tra interno no incluye explícitamente la notación de valor absoluto de las fuerzas, si bien se puede sobrentender de la narración de Otto, pues en sus ejemplos siempre utiliza valores positivos, también para compresiones. Este modo de aplicación hace que la expresión matemática de Tra interno y cantidad de estructura sean idénticas. Ambos grupos de investigación valoran otras posibilidades para la denominación de estos conceptos, llegando incluso a coincidir en la opción de volumen de tensiones.

c) Todas las magnitudes definidas anteriormente tienen unidades físicas precisas: Tra interno, Tra externo y cantidad de estructura se miden en kNm correspondiéndose con el concepto físico de trabajo, y Bic es el cociente entre masa y trabajo, medido en $\mathrm{g} / \mathrm{kNm}$. La esbeltez en pandeo de Ricardo Aroca tiene como unidad $\mathrm{m}^{2} / \mathrm{N}$, quizás más intuitivo que el lambda de Frei Otto que se mide en $\mathrm{m} / \sqrt{ } \mathrm{N}$. Los únicos parámetros adimensionales son el Número de Michell y el Tra relativo, tal como sus nombres indican.

d) Tanto el Tra relativo como el número de Michell miden, para cada modelo propuesto, el rendimiento en el transporte de cargas respecto de un valor de referencia. Sin embargo, este valor de referencia se consigue en la escuela madrileña como el producto de la carga total por la dimensión del problema (la luz en casos de flexión), resultando por definición, un valor mayor o igual al del IL, y por tanto $v \leq$ Tra relativo. Así pues, entre los conceptos de Tra relativo y número de Michell existe una relación 
de proporcionalidad cuya constante es el cociente entre el tamaño de la estructura y la distancia de la carga a los apoyos. Entendiendo que, para problemas con varias fuerzas exteriores, esta distancia es la ponderada, es decir, el sumatorio del producto de cada una de las cargas por su separación al apoyo más cercano (Tra externo) dividido entre el sumatorio de todas las cargas.

e) El concepto de Bic depende de la masa del sistema, y ésta a su vez, para determinados problemas de carga, es función de la esbeltez de la solución. De esta forma Frei Otto introduce las condiciones del material (posiblemente motivado por la búsqueda de la ligereza como fin último) en los criterios de eficiencia. Sin embargo, los profesores de la ETSAM buscan una teoría del consumo estructural más abstracta, independiente del material, por lo que introducen las condiciones de esbeltez en la formulación generalizada de cantidad de estructura, de acuerdo a la expresión [9].

Si varía la esbeltez, la cantidad de estructura también varía, pero de forma directa y sin necesidad de dimensionar para calcular la masa que soporta los esfuerzos producidos (Bic). Con esta operación el concepto inicial de cantidad de estructura aumenta su contenido teórico al hacerse independiente del dimensionado e integrar a la esbeltez en su formulación. La relación entre Bic y los conceptos manejados por este segundo grupo resulta sencilla, pues siendo Bic $=\mathrm{m} / \mathrm{Tra}$, con $\mathrm{Tra}=\mathrm{Ta}=\mathrm{aQl}$ resulta:

$$
\text { Bic }=\frac{\mathrm{m}}{\mathrm{Ta}}=\frac{\mathrm{P}}{\mathrm{gTa}}=\frac{\mathrm{W}}{\frac{\sigma}{\rho} \mathrm{gTa}}=\frac{\gamma \mathrm{Ql} \lambda}{\frac{\sigma}{\rho} \mathrm{g} \alpha \mathrm{Ql}}=\frac{\gamma \lambda}{\alpha}=\frac{1}{\mathrm{Ag}}=\frac{\nu}{\alpha} \mathrm{Bic}_{\text {mat }}
$$

con $\mathrm{Ag}$ igual al producto del alcance del material $\sigma / \rho$ por la aceleración de la gravedad, o inverso del Bic material (masa unitaria partida por resistencia).

Los valores de estos coeficientes se pueden tabular para los distintos modelos estructurales, planteando por lo tanto una cuantificación independiente de la carga. Para la misma carga exterior el modelo más eficiente es el de menor coeficiente de proporcionalidad.

f) Sin embargo, ambos grupos son conscientes de que mediante la introducción de la masa, y por lo tanto la densidad y el peso específico, como parámetro es posible la aplicación de sus conceptos para la determinación de los tamaños límite de objetos sosteniendo únicamente su propio peso. Así, la «longitud de rotura» de Otto y el «alcance» madrileño son el valor inverso del Bic material (cociente entre densidad y tensión de rotura).

g) Sólo Otto y su grupo han tratado de desarrollar el concepto de esbeltez, $\lambda$, desde un enfoque relativo a la geometría y cargas globales. Para el grupo madrileño esta idea se aplica sólo a nivel local o de pieza, y de forma diferente si ésta resiste esfuerzos de compresión o flexión. En flexión la esbeltez es un parámetro puramente geométrico e independiente de las cargas. Para los problemas de pandeo el concepto introducido por Aroca y de Miguel, $\mathrm{l}^{2} / \mathrm{N}$, es equivalente al cociente entre geometría y fuerza del alemán, ya que

$$
\mathrm{l}^{2} / \mathrm{N}=(\mathrm{l} / \sqrt{ } \mathrm{N})^{2}=(\mathrm{s} / \sqrt{ } \mathrm{F})^{2}=\lambda^{2}
$$

\section{APLICACIÓN A ESTRUCTURAS DE GEOMETRÍAS PREFLECTADAS}

El análisis estructural de las estructuras generadas por elementos preflectados debe ser congruente con su proceso constructivo. Partiendo de elementos originalmente planos, y durante un proceso denominado búsqueda de la forma, se introduce paulatinamente una acción exterior en los elementos hasta que se consigue la forma deseada. Una vez preflectados y ensamblados, los elementos estructurales están preparados para soportar las acciones para las que han sido proyectados. Este proceso constructivo conlleva, al menos durante la fase de búsqueda de la forma, la introducción de tensiones internas no despreciables y la aparición de grandes desplazamientos. La no linealidad geométrica y el estado de pretensión anterior a la entrada en carga hacen que la aplicación de los conceptos de eficiencia estructural no sea inmediata. En primer lugar es necesario retornar al origen teórico de estas magnitudes -el volumen de tensiones- (Figura 4) que permitirá, una vez discretizado el continuo, proporcionar soluciones suficientemente aproximadas utilizando el Método de los Elementos Finitos, que incluyan y distingan los efectos de las tensiones derivadas de la búsqueda de la forma y de la entrada en carga de las acciones. En segundo lugar, la presencia de grandes rotaciones y desplazamientos hace indispensable la actualización de los valores de las distintas magnitudes para cada paso de carga, de acuerdo con la modificación de las distancias en la geometría del sistema.

En las estructuras habituales en edificación, sin tensiones previas ni grandes deformaciones, el modo de aplicación de las teorías presentadas consiste en obtener los valores numéricos del concepto utilizado. Posteriormente este valor se compara con los de otras soluciones ya calculadas, o usando los diagramas y tablas publicados (Figuras 1 y 2) para juzgar el consumo estructural. En este trabajo se presenta la aplicación a estructuras con tensiones residuales, por lo que los conceptos se analizan para los sucesivos valores de un factor de carga, y revelar así cómo afecta la variación de las tensiones internas al comportamiento global.

\subsection{Estructuras preflectadas emparejadas}

La explotación del curvado elástico de elementos originalmente planos para la obtención de superficies destinadas al uso de cubierta se remonta, en el caso del mudhif iraquí (Figura 5) o la yurta mongola, a unos seis mil años de antigüedad. Aquí podríamos encontrar una primera indicación de la eficiencia y control del consumo de material de estas estructuras, pues se encuentran tradicionalmente en áreas donde la madera es escasa, o en culturas que no han desarrollado las técnicas propias de la carpintería de grandes escuadrías (11).

Tras un siglo XX con pocos ejemplos de edificios que instrumentalicen la deformación elástica, en estos últimos siete años un grupo de arquitectos e ingenieros alemanes, pertenecientes como Otto a la Universidad de Stuttgart, han renombrado -flexión activa- y rescatado este método constructivo para incluir las propiedades del material en los protocolos informatizados de la búsqueda de la forma y así obtener un mayor control sobre la forma final obtenida. Dentro de las estructuras proyectadas por este grupo tiene especial interés el pabellón ICD/ITKE 2010, pues está formado por una serie de elementos radiales que se entrelazan con los dos más próximos formando arcos complejos por sucesión de parejas arco-tirante (Figura 5).

La división más elemental de las estructuras preflectadas pasa por la clasificación según el modo en el que se consiguen los esfuerzos de preflectado. En este sentido podemos 

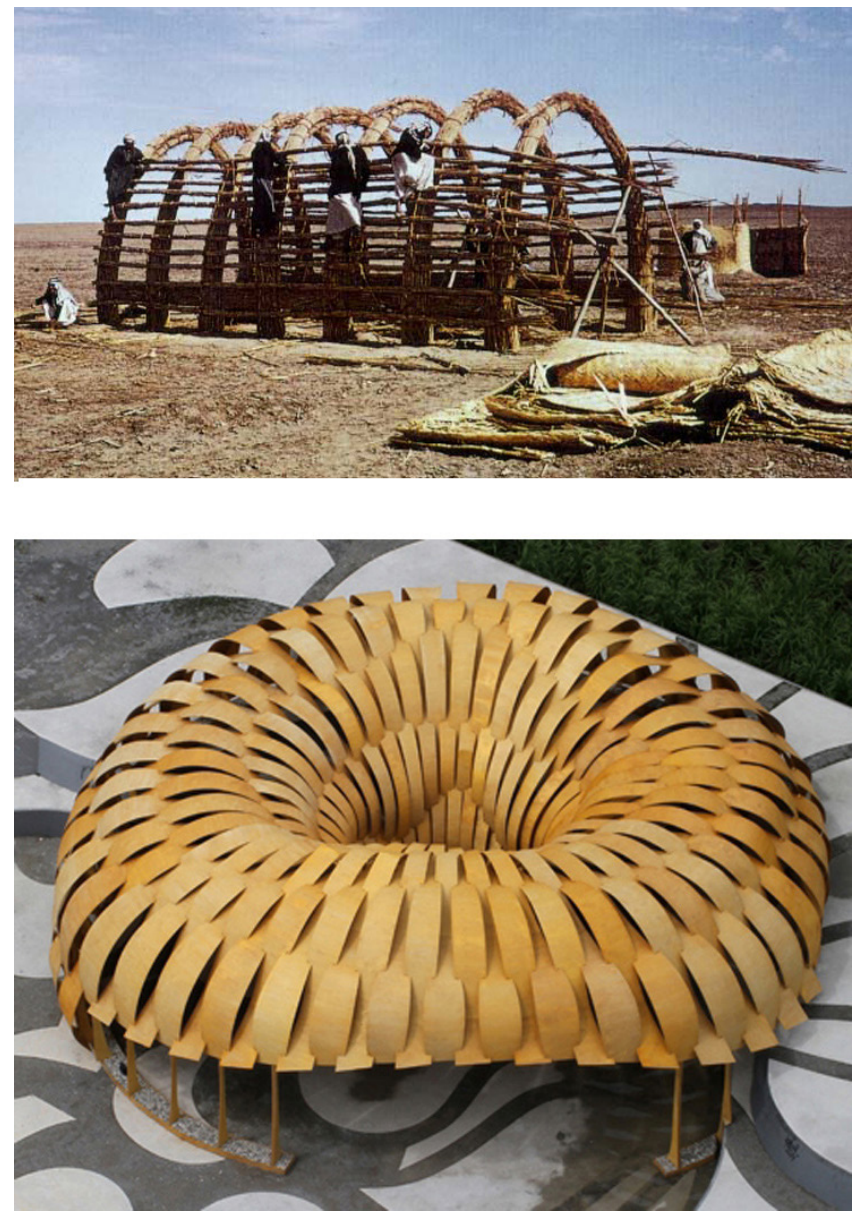

Figura 5. Mudhif iraquí en construcción. Fuente: (12). Pabellón temporal ICD/ITKE 2010. Fuente: (14).

separar entre las estructuras que producen o necesitan de un empuje en los apoyos y aquellas que por su conformación se encuentran en equilibrio respecto de los esfuerzos introducidos para provocar la deformación, sin generar reacciones exteriores. Este equilibrio sólo es posible mediante el emparejamiento de elementos, de tal forma que uno se encuentre comprimido y otro traccionado.

Como ejemplo de estructura emparejada se ha escogido la presentada por Lienhard et al. (13) para explicar el funcionamiento estructural del pabellón ICD/ITKE 2010. La construcción del modelo se realiza a partir de dos listones, uno relativamente más largo que el otro. Mediante la contracción de unos tensores auxiliares se consigue poner las testas de ambos extremos en los mismos planos, obteniendo así la forma final (Figura 6). En este proceso de búsqueda de la forma se introducen tensiones en los elementos estructurales. Tradicionalmente a estas tensiones se las denomina esfuerzos residuales.

\subsection{Método de análisis y aplicación de los conceptos}

Para reflejar simultáneamente la potencia de los conceptos de consumo estructural y de las estructuras emparejadas se ha realizado la comparación de dos estructuras distintas (A y B), pero que utilizan el mismo material y volumen estructural, y, por lo tanto, con la misma masa. La diferencia radica en que tan sólo la solución A incorpora los esfuerzos residuales. La solución B es la referencia geométrica de la primera, pues

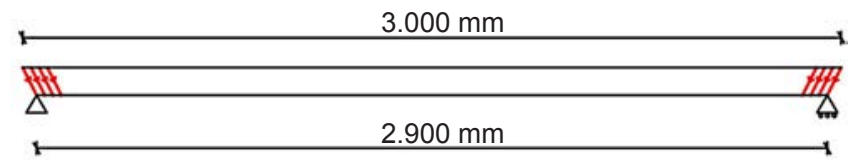

FASE 0

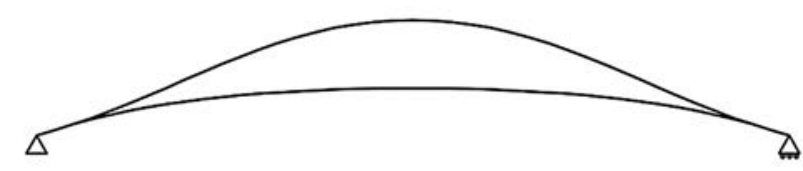

FASE 1

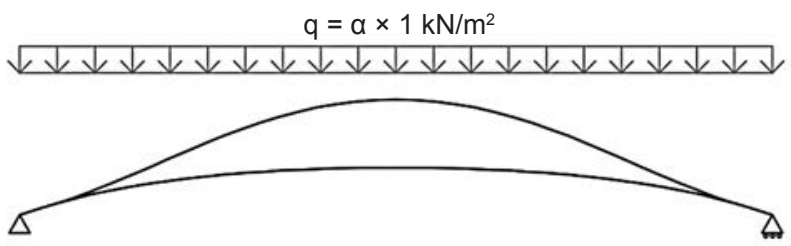

FASE 2

Figura 6. Estructura preflectada emparejada. Fase o: Estado inicial con los tensores. Fase 1: Búsqueda de la forma. Fase 2: Introducción carga exterior.

tiene la misma forma pero no tiene en cuenta los esfuerzos necesarios para su obtención. Más allá de esta diferencia, el proceso de análisis es idéntico para ambas estructuras y consta de dos fases: una primera fase de búsqueda de la forma mediante el incremento del pretensado de los tensores, y una segunda en la que se introduce la carga exterior de forma progresiva.

$\mathrm{Al}$ tratarse de estructuras conformadas por elementos muy deformables en una dirección, la forma se obtiene mediante un proceso iterativo de deformaciones elásticas, o de segundo orden. Esta carencia de linealidad determina los resultados, por lo que se ha incorporado - para los conceptos y casos en los que la distancia a apoyos se modifica sustancialmente- un valor adicional entre paréntesis resultado de aplicar las dimensiones del sistema inmediatamente posteriores al proceso de búsqueda de la forma, en comparación con los valores actualizados para la última deformada -cifra sin paréntesis-.

Para conocer la influencia del sentido de la carga en el comportamiento estructural se analiza cada una de las soluciones para dos factores de carga, $\alpha$. El valor $\alpha=1$ representa la carga en sentido descendente, mientras que $\alpha=-\mathrm{s} 1$ indica sentido de carga ascendente.

Además, con el fin de valorar únicamente la repercusión de las acciones exteriores, en la Tabla 1 se ha dividido la columna de la propuesta que tiene en cuenta las tensiones residuales, para cada factor de carga, en otras dos columnas. La primera incluye los valores correspondientes con las tensiones actualizadas para la deformada final, $\sigma_{\mathrm{d}}$. La segunda entrada, $\Delta \sigma$, es el resultado de restar a las tensiones finales las tensiones introducidas durante el proceso de búsqueda de la forma, o tensiones residuales, $\sigma_{\mathrm{bf}}$.

\section{5•3. Resultados}

La relación de proporcionalidad existente entre el Número de Michell y Tra relativo se mantiene especialmente para la entra- 
Tabla 1. Tabla comparativa de las dos soluciones estudiadas.

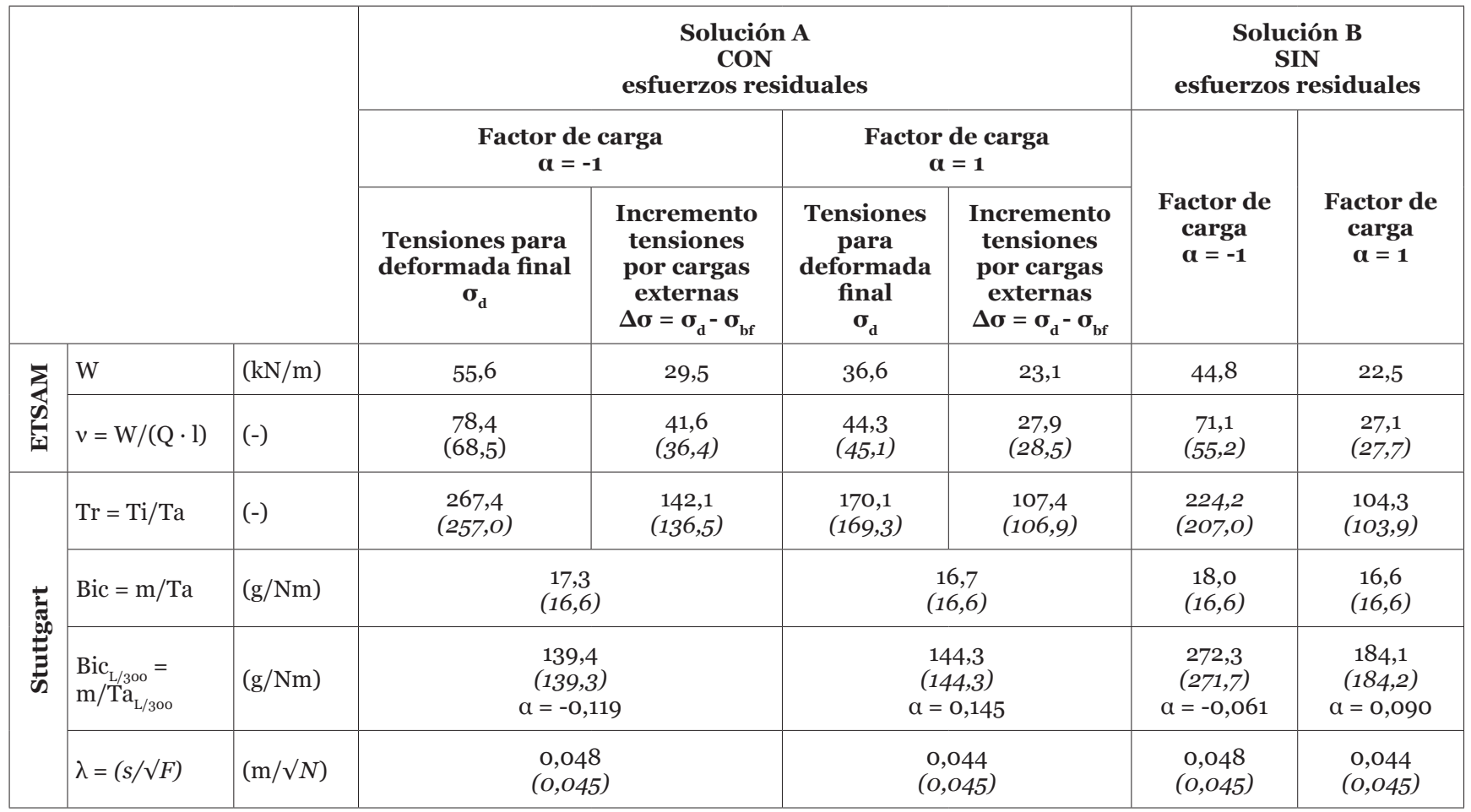

Valor resultado de aplicar las dimensiones del sistema actualizadas para la última deformada.

(Valor resultado de aplicar las dimensiones del sistema tras el proceso de búsqueda de forma).

da en la que, para medir en exclusiva la respuesta estructural frente a las cargas exteriores, se reducen las tensiones resultantes mediante la sustracción de las obtenidas en el proceso de la obtención de la forma. Este invariante valida la operación de reducción de tensiones y hace evidente la coherencia de los conceptos de eficiencia de ambos grupos de investigación.

En la Tabla 1 se incorporan dos valores distintos de Bic. El primero, que se corresponde con el factor de carga aplicado y no con la carga de rotura que sería lo habitual, sirve para mostrar que la masa utilizada para las dos soluciones es la misma. Las pequeñas variaciones numéricas son consecuencia de las diferencias formales de las deformadas finales. Sin embargo, y para introducir los criterios de rigidez en la medición de la eficiencia, el segundo valor de Bic es el asociado con una deformación determinada, en este caso para L/300. Esta adaptación sirve para demostrar que la solución que incorpora los esfuerzos residuales es más eficiente, pues para la misma flecha provocada por las cargas exteriores multiplicadas por un factor de carga $\alpha=$ - 1 consume un $49 \%$ menos de material que la estructura con la misma geometría, pero sin tensiones de formación.

Este aumento de rigidez viene producido porque la configuración de la carga externa somete a compresión al tirante originalmente traccionado, que se ve progresivamente descargado de su esfuerzo normal (14). Si realizamos las gráficas (Figura 7) con los distintos valores de Número de Michell y Tra relativo de ambas estructuras para los distintos factores de carga de las acciones propuestas y nos fijamos en su modo de crecimiento, se observa que la curva de la estructura que tiene en cuenta los esfuerzos residuales consta de dos partes diferenciadas, delimitadas por el punto con tangente a $45^{\circ}$, para el factor de carga $\alpha=-0,8$. Para factores de carga $0>\alpha$ $>-0,8$, el tirante todavía se encuentra traccionado, mientras que para factores $\alpha<-0,8$ empieza a comprimirse. Si compa- ramos este segundo tramo se observa que es sensiblemente paralelo para las tres curvas, revelando un comportamiento estructural similar una vez que desaparecen los efectos positivos del preflectado.

Sin embargo, las mayores diferencias se observan si comparamos las soluciones en el tramo para $0>\alpha>-0,8$ mientras el tirante se encuentra traccionado. La eficiencia se puede medir directamente como el inverso del Número de Michell o del Tra relativo cuando las tensiones iniciales son nulas. En el caso de contar con tensiones de conformación, no tiene sentido hablar de la eficiencia de un esquema estructural que ya tiene esfuerzos para una carga exterior nula. La solución inicial que aquí se plantea es la comparación directa con los valores de la curva que sólo tiene en cuenta los efectos de las cargas externas (sustracción de las tensiones residuales). Pero además podemos medir la eficiencia utilizando la variación de estas magnitudes para los mismos incrementos de carga. El proceso de análisis de estas estructuras, de introducción de las acciones mediante pasos de carga, permite apreciar esta característica con claridad. Una menor pendiente -respecto del eje que mide $v$ y Tr- de la curva implica que para el mismo incremento de carga las tensiones internas aumentan a mayor velocidad, y por lo tanto $v$ y Tr, disminuyendo la eficiencia. Se puede apreciar que, independientemente de si se utiliza $v$ o Tr, para el intervalo o $>\alpha>-0,8$, la curva que sólo tiene en cuenta el incremento de las tensiones debidas a las cargas externas tiene mayor pendiente y, por lo tanto, mayor eficiencia respecto de la variación de carga que la estructura sin tensiones residuales.

\section{CONCLUSIONES}

El principal beneficio de la inclusión de estos conceptos de cuantificación de consumo estructural en las fases iniciales 


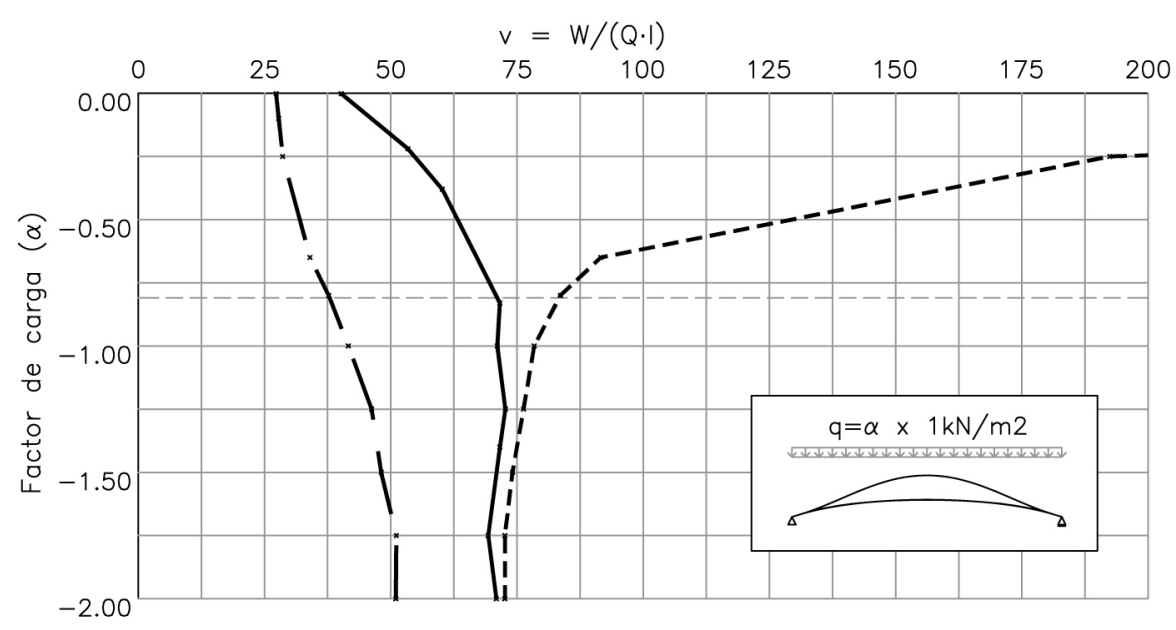

Esfuerzo normal en cordón inferior (N)
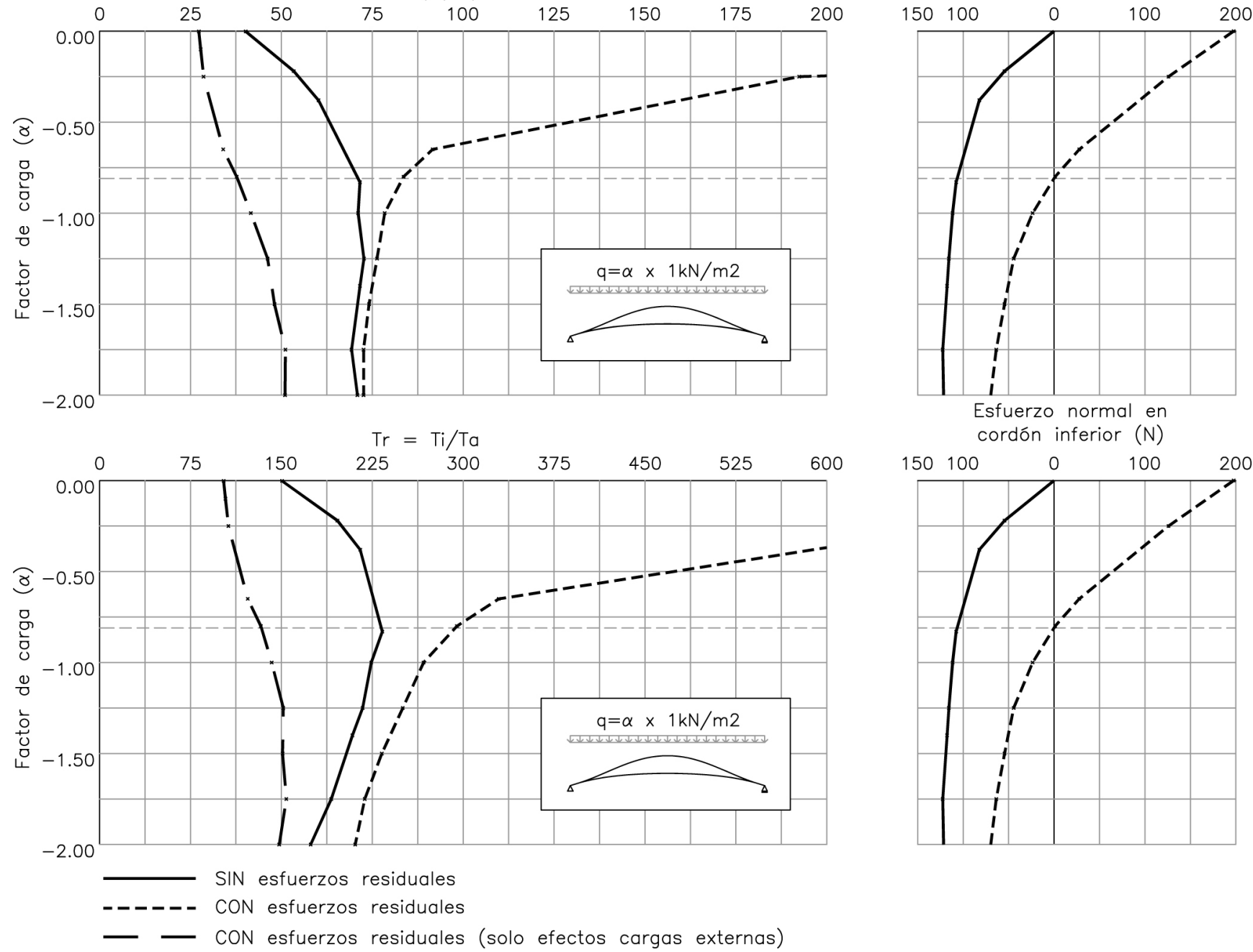

Figura 7. Comparación de los valores del Número de Michell y del Tra relativo de la estructura emparejada para distintos factores de carga.

del proyecto de estructuras es que permiten cualificar las distintas opciones posibles. De esta manera se pueden realizar comparaciones objetivas y rigurosas para determinar cuál es la mejor -bondad-solución, por encima del clásico concepto de eficiencia como la relación entre carga útil y carga total (incluyendo el propio peso).

La coincidencia en el propósito de las dos teorías presentadas, medir el consumo estructural, hace que sus conceptos base (Tra y cantidad de estructura) sean matemáticamente idénticos. Para evaluar la eficiencia del sistema que se quiere estudiar estos conceptos se relacionan con los valores de referencia del problema que intentan resolver. Consecuentemente, los resultados numéricos que se obtienen al realizar esta operación son proporcionales, como se ha podido comprobar en el ejemplo analizado. Sin embargo, los dos grupos afrontan la inclusión de la masa de forma distinta: Otto la incorpora para crear un nuevo concepto que mida la ligereza y la eficiencia del esquema simultáneamente, mientras que el grupo de profesores de la ETSAM prefiere independizar el parámetro de medida de eficiencia de la forma respecto de las cualidades del material.

En este sentido la tabla Tra (Figura 1) y el diagrama Bic (Figura 2) suponen la demostración particular de lo que en la Escuela de Madrid se ha obtenido de forma teórica a través de la formulación de teoremas, y viceversa: los esquemas más eficientes tienen esfuerzos de igual signo o mantienen sus barras ortogonales antes y después de la deformación (8). Las diferencias de los valores de la tabla Tra y el resultado de aplicar el concepto de cantidad de estructura -para el mismo esquema estructural- son mínimas: para soluciones ya comparadas y una vez modificados por el factor de proporcionalidad se obtienen diferencias menores del $5 \%$. Esta variación se puede justificar con las afirmaciones del propio Frei Otto que califica los cálculos como aproximados al haber sido realizados mediante métodos gráficos.

Ambas teorías se aplican de la misma forma: multiplicando fuerzas por distancias se obtiene el valor aritmético del concepto para el esquema estructural. Este valor se compara con los valores obtenidos por los distintos diseños geométricos que se están estimando. De esta comparación numérica se deduce la opción más eficiente. Pero si las estructuras cuentan con tensiones previas -originadas, por ejemplo, durante el preflectado-, estos conceptos valen, además, para realizar comparaciones del esquema para distintos pasos de carga, y descubrir las distintas fases de comportamiento estructural. El campo de aplicación ideal de la estructura será aquel en el que las cargas de servicio corresponden con los pasos de carga en los que la estructura se comporta de una forma más eficiente. 
En el ámbito de toma de decisiones en el diseño de estructuras preflectadas se han de considerar los efectos en la rigidez de la estructura derivados de la forma y de las tensiones residuales. Estos efectos del preflectado pueden ser positivos, negativos o despreciables, y deben estudiarse para cada caso particular (15) (16). Las estructuras preflectadas pueden, si se las compara con soluciones tradicionales con el mismo consumo de material, reducir la flecha como resultado de la consecución de formas más eficientes (17). Además al com- pararlas con su referente geométrico, tal y como se ha visto en el ejemplo, se observa que se puede incrementar la rigidez mediante la inducción durante el preflectado de esfuerzos normales de tracción que se descarguen para las hipótesis de carga de servicio. Para estas condiciones de diseño los valores de los conceptos analizados de las soluciones con tensiones residuales y sin ellas tienden a igualarse, mientras que las deformaciones son menores para las soluciones preflectadas.

\section{REFERENCIAS}

(1) Fernández, J. L. (1998). Estructura: tamaño, forma y proporción (Tesis doctoral). Madrid: Escuela Técnica Superior de Arquitectura, Universidad Politécnica de Madrid.

(2) Rankine, W. J. M. (1863). A Manual of Civil Engineering, London: Griffin Bohn and Co.

(3) Maxwell, J. C. (1890). Reciprocal figures, frames, and diagrams of forces. Scientific Papers, vol. II, pp. 175-177. Cambridge University Press.

(4) Michell, A. G. M. (1904). The limits of economy of materials in frame-structures. Philosophical Magazine, 8(47): 589597.

(5) Otto, F. (1998). IL 24 - Lightweight principle, Stuttgart: Institute for Lightweight Structures.

(6) Miguel, J. L. de. (1974). El trabajo estructural, un nuevo escalar de las estructuras (Tesis doctoral). Madrid: Escuela Técnica Superior de Arquitectura, Universidad Politécnica de Madrid.

(7) González, J. A. (1990). Análisis del proceso de diseño de estructuras porticadas (Tesis doctoral). Madrid: Escuela Técnica Superior de Arquitectura, Universidad Politécnica de Madrid.

(8) Cervera, J. (2011). Concebir y analizar estructuras. Madrid: Departamento de Estructuras de la Edificación, Escuela Técnica Superior de Arquitectura, Universidad Politécnica de Madrid.

(9) Cervera, J., Ortiz, J., Vázquez Espí, M., Aznar, A. (2013). Dimensionado en compresión en acero: el peso del pandeo. Revista Internacional de Métodos Numéricos para Cálculo y Diseño en Ingeniería, 29(2): 79-91, doi: https://doi. org/10.1016/j.rimni.2013.04.005.

(10) Cervera, J., Vázquez, C., Vázquez M. (2014). Two near-optimal layouts for truss-like bridge structures bearing uniform weight between supports. Journal of Structural Engineering, 140(4). American Society of Civil Engineers.

(11) Lienhard, J., Alpermann, H., Gengnagel C., Knippers, J. (2013). Active bending, a review on structures where bending is used as a self-formation process. International Journal of Space Structures, 28(3\&4), doi: http://dx.doi. org/10.126o/0266-3511.28.3-4.187.

(12) Ochsenschlager, E. (1998). Life on the edge of the marshes. Expedition 40(2): 29-40. Philadelphia: University of Pennsylvania, Museum of Archaelogy and Anthropology.

(13) Lienhard, J., Schleicher, S., Knippers, J. (2011). Bending-active Structures - Research Pavilion ICD/ITKE. En Nethercot, D., Pellegrino, S. et al. (Eds.), Taller, longer, lighter. Proceedings of the International Symposium of the IABSEAISS Symposium, London, UK.

(14) Lienhard, J. (2014). Bending-Active Structures (Tesis doctoral). Stuttgart: Universität Stuttgart - Institut für Tragkonstruktionen und Konstruktives Entwerfen.

(15) Gengnagel, C., Alpermann, H., Lafuente, E. (2014). Active bending in hybrid structures. En Filz, G. H., Maleczek, R., Scheiber, C. (Eds.), FORM - RULE $\mid$ RULE - FORM 2013 (pp. 12-27). Innsbruck University Press.

(16) Schleicher, S., Rastetter, A., La Magna, R., Schönbrunner, A., Haberbosch, N., Knippers, J. (2015). Form-finding and design potentials of bending-active plate structures. En Modelling Behaviour (pp. 53-63). Springer International Publishing, doi: https://doi.org/10.1007/978-3-319-24208-8_5.

(17) García, A., Monjo, J., Sakata, H., Sastre, R., Yamazaki, Y. (2016). Actively-bent plywood floor frame with in-plane curvature. En Kawaguchi, K., Ohsaki, M., Takeuchi, T. (Eds.). Spatial Structures in the 21st Century. Proceedings of the International Symposium of the IASS 2016, Tokyo, Japan, 2016.

(18) Samyn, P., Latteur, P. (2000). Volume and displacement indicators of isostatic structures the case of the horizontal isostatic span, vertically loaded. En Bridge between civil engineering and architecture. Proceedings of the $4^{\text {th }}$ International Colloquium on Structural Morphology. Delft: Delft University of Technology. 